\title{
Erratum to: Smale's Fundamental Theorem of Algebra Reconsidered
}

\author{
Diego Armentano - Michael Shub
}

Published online: 14 June 2014

(C) SFoCM 2014

\section{Erratum to: Found Comput Math (2014) 14:85-114 DOI 10.1007/s10208-013-9155-y}

The proof of Lemma 11-(ii), for $p>1$ is incorrect. Consequently:

1. Theorem 3 is unproven.

2. Theorem 2 is incorrect for $p>1$.

3. The assertion of the introduction that the homotopies

$$
f_{t}=f-(1-t) \Delta\left(\frac{\langle\cdot, \zeta\rangle^{d_{i}}}{\langle\zeta, \zeta\rangle^{d_{i}}}\right) f(\zeta)
$$

lead to an average polynomial time algorithm to find approximate zeros for $f \in$ $\mathcal{H}_{(d)}$ when the Bézout number is polynomial in the input dimension, including the case $n=1$ and $D>n^{1+\varepsilon}$, is unproven.

4. The integral in footnote 1 is infinite. The proof is like Lemma 11-(i).

The online version of the original article can be found under doi:10.1007/s10208-013-9155-y.

\section{Armentano}

Centro de Matemática, Universidad de la República, Iguá 4225, Montevideo 11400, Uruguay e-mail: diego@cmat.edu.uy

M. Shub $(\varangle)$

CONICET, IMAS, Universidad de Buenos Aires, Ciudad Universitaria,

Buenos Aires C1428EGA, Argentina

e-mail: shub.michael@gmail.com 